\title{
Lian Gong em 18 terapias: uma proposta para prevenir os transtornos traumáticos cumulativos
}

Recebido em: 14/07/2012

Aceito em: 30/01/2013

\author{
Maria Rosilene Cândido Moreira' \\ Fernanda Leite Dias ${ }^{2}$ \\ Alda Maria da Silva ${ }^{3}$ \\ Adenusca Suérica Alencar de Souza ${ }^{4}$ \\ Laryssa Ramos Beserra 5 \\ Jefferson Gomes de Abreu ${ }^{5}$ \\ Paloma Lopes de Asevedo ${ }^{5}$ \\ Milena Gabriela dos Santos Silva ${ }^{5}$
}

Transtornos traumáticos cumulativos incapacitam para o trabalho e as práticas corporais chinesas podem preveni-los quando praticadas regularmente. Este artigo relata experiências de acadêmicos de enfermagem na elaboração do projeto para inserção do Lian Gong na rotina de trabalhadores. Foram 12 reuniões em maio de 2012 para definição do arcabouço do projeto e realização dos exercícios. Inicialmente houve timidez pela exposição corporal e preocupação em acertar os movimentos. Entretanto, praticando o Lian Gong, o grupo percebeu mudanças positivas, como bem-estar, redução da timidez e maior integração. Essa experiência enriqueceu o aprendizado e já apresentou resultados satisfatórios quando praticada pelos proponentes.

Descritores: Transtornos Traumáticos Cumulativos, Medicina Tradicional Chinesa, Saúde do Trabalhador.

\section{Lian Gong in 18 therapies: a proposal for preventing cumulative traumatic disorders}

Cumulative traumatic disorders make a person unable to work and the Chinese body practices can prevent their occurrence when practiced regularly. This article describes experiences from university students of Nursing in the preparation of the project for introducing the Lian Gong practice in the routine of workers. 12 meetings were held in May 2012 to define the framework of the project and performance of the exercises. Firstly, there was some shyness because of body exposure and concern with getting the movements right. However, after having practiced Lian Gong, the group could notice some positive changes such as well-being, decreased shyness, and increased integration. Such experience enriched the learning and presented satisfactory results when practiced by the proponents.

Descriptors: Cumulative Traumatic Disorders, Chinese Traditional Medicine, Occupational Health.

\section{Lian Gong en 18 terapias: una propuesta para prevenir los trastornos traumáticos cumulativos}

Trastornos traumáticos cumulativos incapacitan para el trabajo y las prácticas corporales chinas pueden prevenirlos cuando practicadas regularmente. Este artículo relata experiencias de académicos de enfermería en la elaboración del proyecto para inserción del Liang Gong en la rutina de los trabajadores. Fueron 12 reuniones en mayo del 2012 para definición del marco del proyecto y la realización de los ejercicios. Inicialmente hubo timidez por la exposición corporal y preocupación en acertar los movimientos. Sin embargo, practicando el Liang Gong, el grupo sintió cambios positivos, como bienestar, reducción de la timidez y mayor integración. Esta experiencia enriqueció el aprendizaje y ya presentó resultados satisfactorios cuando practicada por los proponentes. Descriptores: Trastornos Traumáticos Cumulativos, Medicina Tradicional China, Salud del Trabajador.

\section{INTRODUÇÃO}

N o tocante aos estudos e práticas sobre as questões pautadas à saúde e ao trabalho, as Lesões por Esforço Repetitivo (LER), atualmente denominadas Distúrbios Osteomusculares Relacionados ao Trabalho (DORT), compõem o conjunto de doenças que mais frequentemente afetam trabalhadores no exercício de suas atividades. Neste grupo estão as afecções que podem acometer tendões, músculos, nervos, fácias e ligamentos, de forma isolada ou associada, com ou sem degeneração de tecidos, atingindo, principalmente, os membros superiores, a região escapular e o pescoço, com origem ocupacional. Frequentemente causam incapacidade temporária ou permanente ${ }^{(1)}$.

Vários fatores associados ao trabalho concorrem para a ocorrência de LER/DORT, como repetição de movimentos, manutenção de posturas inadequadas, esforço físico excessivo, invariabilidade de tarefas, pressão mecânica sobre

1 Enfermeira. Mestre em Saúde Coletiva. Professora Assistente da Universidade Federal de Campina Grande (UFCG). Membro do Grupo de Pesquisa em Saúde Coletiva (GPESCUFCG). Coordenadora do projeto Utilização do Lian Gong em 18 terapias na prevenção de LER/DORT (PROEXT-MEC-SESU/PROBEX-UFCG). E-mail: rosilenecmoreira@gmail.com 2 Acadêmica de Enfermagem da UFCG. Bolsista do Programa de Extensão Universitária do Ministério da Educação (PROEXT-MEC/SESu).

3 Acadêmica de Enfermagem da UFCG. Bolsista do Programa de Bolsas de Extensão Universitária da UFCG (PROBEX/UFCG).

4 Acadêmica de Enfermagem da UFCG. Membro do GPESC/UFCG.

5 Acadêmica de Enfermagem da UFCG. 
determinados segmentos do corpo, trabalho muscular estático, impactos e vibrações. A intensificação do ritmo, da jornada e da pressão por produção, assim como a perda acentuada do controle sobre o processo de trabalho por parte dos trabalhadores (fatores relacionados à organização do trabalho), têm sido apontados em diversos estudos como os principais determinantes de LER/DORT ${ }^{(2,3)}$, condições que desencadeiam dores crônicas, fadiga muscular e inflamações nas articulações.

Observamos as LER/DORT como quadros inicialmente inflamatórios e de fadiga músculo-esquelética, resultantes da rapidez e excesso de movimentos, manutenção de determinadas posturas por tempo prolongado e falta de tempo para recuperação. Inicialmente são relatados dores e formigamentos de forma difusa, mas pouco intensas; posteriormente, ocorre a cronificação dos sintomas e a evolução para quadros neurológicos e de alterações no sistema nervoso autônomo, extremamente incapacitantes ${ }^{(4)}$.

Por estarem diretamente ligadas à atividade desenvolvida pelo trabalhador e ainda provocarem perda ou diminuição permanente ou temporária da capacidade funcional para o exercício laboral, as LER/DORT estão classificadas como acidentes de trabalho, devendo ser imediatamente notificadas ao setor de segurança do trabalho da empresa, logo que o trabalhador tenha o diagnóstico estabelecido ${ }^{(5)}$.

Sob esse prisma, as LER/DORT podem levar o trabalhador a afastar-se das suas atividades laborais temporária ou permanentemente, dependendo da gravidade do problema que se apresenta. $O$ tratamento a ser escolhido e os seus resultados estão relacionados tanto ao grau da lesão, como também a um diagnóstico correto, à eliminação dos agentes causadores ou agravantes e a uma estratégia terapêutica multiprofissional, na qual devem ser encorajadas propostas que envolvam o tratamento, a prevenção de complicações e a promoção da saúde no ambiente de trabalho.

A percepção dos limites do corpo, a postura adequada para realizar atividades, a combinação da terapêutica medicamentosa (e em alguns casos, a intervenção cirúrgica), com as terapias não farmacológicas tais como massagens, hidroginástica e técnicas de relaxamento podem, a curto e longo prazo, minimizar os prejuízos causados na vida dos trabalhadores ${ }^{(2)}$.

Direcionando-se à promoção da saúde do trabalhador, com a implantação do Sistema Único de Saúde (SUS) no país, a atenção primária à saúde passou por várias modificações no que diz respeito ao processo assistencial desta parte da população, com a implementação de práticas integrativas e complementares que visam a obtenção de resultados favoráveis sobre os indicadores de saúde no mundo do trabalho(6).

Partindo da premissa de que o estado de saúde relacionado ao trabalho corresponde a um estado de equilíbrio entre corpo, mente e espiritualidade em harmonia com o ambiente laboral, e que as doenças ocupacionais são vistas como uma ruptura dessa harmonia, pois comprometem as funções do organismo ${ }^{(7)}$, as práticas complementares corporais da medicina tradicional chinesa têm sido progressivamente utilizadas no tratamento e prevenção das LER/DORT ${ }^{(8,9)}$. Isso porque elas se baseiam na interação entre a manutenção da saúde e a prevenção da doença, buscando harmonizar o estado de saúde geral das pessoas.

Nesse contexto, vem se destacando a prática do Lian Gong em 18 terapias, por apresentar-se como um método que exige apenas a tecnologia leve para sua execução, sendo aplicável tanto a trabalhadores como ao público que assim o desejar.

O Lian Gong em 18 terapias foi criado na década de 1960, pelo médico ortopedista chinês Zhuang Yuen Ming. O mesmo embasou-se em técnicas milenares como o Do-In, o Tui-Na, o jogo dos cinco animais e o exercício dos oito brocados de seda, para criar esta ginástica terapêutica de prevenção e tratamento de problemas músculo-esqueléticos. Dessa forma, conseguiu preservar aspectos das práticas tradicionais chinesas, facilitando seu aprendizado e viabilizando o autotratamento ${ }^{(10)}$.

No Brasil, a prática do Lian Gong em 18 terapias foi introduzida em 1984 pela professora da Universidade de Campinas, Maria Lucia Lee, que há algum tempo realiza estudos acerca do ensino da filosofia das artes corporais chinesas. No ano de 2006, o Ministério da Saúde incluiu o Lian Gong como um componente das práticas integrativas

e complementares autorizadas através da Política Nacional de Práticas Integrativas e Complementares no SUS ${ }^{(11)}$ como prática de prevenção, promoção e reabilitação em saúde ${ }^{(12)}$.

O Lian Gong é composto de 54 exercícios divididos em três partes, onde a primeira previne e trata dores no pescoço, ombros, costas superiores, região lombar e membros inferiores (Lian Gong Qian Shi Ba Fa); a segunda envolve articulações, tendões e órgãos internos (Lian Gong Hou Shi Ba Fa) e a terceira atua nos distúrbios das vias respiratórias e cardiovasculares (Lian Gong Shi Ba Fa Xu Ji), sendo caracterizada ainda como uma atividade de promoção da saúde, na medida em que garante o desenvolvimento das habilidades pessoais, da qualidade de vida e proporciona uma discussão e construção do conceito de saúde ${ }^{(13)}$.

Como cada parte é subdividida em três séries de 18 exercícios cada uma, o Lian Gong recebe a denominação de Lian Gong em 18 terapias (Lian Gong Shi Ba Fa), cujos movimentos são de fácil assimilação, com duração de, em média, 12 minutos diários, tratando e prevenindo a maioria dos problemas decorrentes de má postura ou de movimentos agressivos à lógica do corpo. Os exercícios, executados em pé, dispensam o uso de roupas especiais para sua prática, sendo acompanhados de música 
exclusiva para a realização das manobras em um ambiente tranquilo para facilitar a apreciação da atividade ${ }^{(14)}$.

Compreendendo o Lian Gong em 18 terapias como medida terapêutica aplicável na prevenção e tratamento das LER/DORT e com foco na promoção da saúde coletiva(15), optamos por utilizar essa prática corporal junto a um grupo de trabalhadores de uma distribuidora de cosméticos situada no sertão paraibano. Visávamos promover o fortalecimento musculo-esquelético desses profissionais nas atividades de esforço repetitivo, conferir os benefícios do Lian Gong em 18 terapias sobre os determinantes de vida e trabalho deste grupo, e assim contribuir para a difusão das práticas integrativas e complementares do SUS nos diversos segmentos sociais.

Nessa perspectiva, direcionou-se o presente relato de experiência, a fim de divulgar a aplicabilidade das práticas integrativas/complementares no campo da saúde do trabalhador, através da elaboração de uma proposta de extensão universitária de natureza terapêutica corporal chinesa que prevê impactos positivos sobre a qualidade de vida dos seus praticantes.

\section{METODOLOGIA}

O presente trabalho, de caráter descritivo, do tipo relato de experiência vivenciada por sete acadêmicos do curso de graduação em enfermagem da Universidade Federal de Campina Grande (UFCG) na elaboração de uma proposta de inserção do Lian Gong na rotina laboral de um grupo de trabalhadores do sertão paraibano, é resultado da elaboração do projeto de extensão universitária intitulado: "Utilização do Lian Gong em 18 terapias na prevenção de LER/DORT".

A proposta que se seguiu e que está apresentada neste artigo teve como foco principal o planejamento de estratégias de ação preventiva e de promoção da saúde de cunho extensivo, objetivando assim a construção de saberes e hábitos saudáveis que agreguem valor à qualidade de vida dos trabalhadores.

Este projeto encontra-se em execução desde o mês de maio de 2012, com término previsto para dezembro deste ano, sendo integrante de uma proposta extensiva maior, fruto das concepções teórico-filosóficas de um grupo de docentes pertencentes ao campo da saúde coletiva da UFCG/ Campus de Cajazeiras. Por objetivarem o retorno social dos conhecimentos científicos produzidos no âmbito acadêmico, tornaram possível a elaboração do Programa de Extensão intitulado Cidadania, Práticas Educativas e Promoção da Saúde no Semiárido Paraibano (CIPEPS).

O Programa CIPEPS, aprovado no Edital No04 do Programa de Extensão Universitária do Ministério da Educação (PROEXT 2011- MEC/SESu), objetiva difundir conhecimentos sobre questões de saúde humana e ambiental a partir de eixos temáticos sobre educação, promoção da saúde e cidadania.

\section{RESULTADOS E DISCUSSÃO}

Para instrumentalizar nosso trabalho, estabelecemos inicialmente um cronograma de atividades, estruturado em quatro encontros diários, para definir os objetivos da proposta, o público-alvo e as ações a serem implementadas, compreendendo assim o arcabouço do projeto e sua logística.

Nesses encontros, surgiram dúvidas e questionamentos acerca das ações que seriam realizadas, o perfil do público mais vulnerável às LER/DORT, o tempo de execução do projeto, a pactuação com a instituição que seria contemplada, os objetivos e metas, a promoção de práticas educativas através de exercícios oriundos dos povos orientais, o despertar da consciência críitica dos trabalhadores sobre a questão do esforço repetitivo, bem como a difícil tarefa de estimular a mudança comportamental e implementação de novos hábitos de vida relacionados ao trabalho.

Fase 1 - fundamentação teórica para execução da proposta Depois de traçados os objetivos e o público, passamos a procurar literatura científica que fundamentasse melhor nossa proposta. Contudo, ao consultar as bases de dados eletrônicos tais como a Scientific Eletronic Library Online (SciELO), a Biblioteca Digital de Teses e Dissertações da Universidade de São Paulo e o Portal de Periódicos da Capes, constatamos que os resultados foram incipientes.

Entretanto, mesmo não encontrando quantidade significativa de produções científicas relacionando o Lian Gong ao tratamento e prevenção das LER/DORT, os achados preocuparam sobremaneira o grupo. Isso porque foram encontrados trabalhos que exprimiam a problemática em tela e a necessidade de medidas que promovessem a melhoria da qualidade de vida desses profissionais, impulsionando--nos a buscar medidas proativas de mudanças desse cenário.

\section{Fase 2 - definição de instrumentos de coleta de dados para situação diagnóstica}

No decorrer do levantamento bibliográfico, selecionamos vários instrumentos de coleta de dados, gerais e específicos, que serão aplicados antes e após os encontros com os trabalhadores, para melhor retratar os efeitos do Lian Gong em 18 terapias sobre os problemas relacionados à saúde desses profissionais, tais como presença/redução/desaparecimento de sintomas osteomusculares, fadiga e dor relacionadas aos esforços repetitivos e mudanças na qualidade de vida.

\section{Fase 3 - exercitando o Lian Gong em 18 terapias}

Passamos a realizar três encontros semanais em um ambiente da UFCG para assistir aos vídeos com os exercícios e praticá-los, a fim de aprimorar a técnica e promover maior integração entre os membros do grupo. 
No primeiro encontro, sentimo-nos intimidados pela presença do outro, pelas expressões gestuais, pela música e o movimento dos corpos, ao mesmo tempo em que nos preocupávamos e nos esforçávamos para manter a postura correta e não errar os movimentos.

Com os encontros regulares e a prática do Lian Gong, percebemos que diversas modificações ocorreram em nosso cotidiano. Por exemplo, a sensação de bem-estar físico, mental e emocional, maior integração entre os participantes do grupo, aumento da motivação e disposição para as atividades acadêmicas, controle do estresse e melhoria da autoestima, reafirmando os achados na literatura científica revisada.

Além disso, o início da prática do Lian Gong pelo grupo despertou em outros alunos e também em alguns docentes a curiosidade pelo tema e, tendo recebido esclarecimentos, começaram a pedir para fazer parte do projeto, situações estas que deram mais disposição ao grupo para continuar.

Vale ressaltar ainda que, com o aprofundamento dos estudos acerca da temática e nosso aprimoramento nos exercícios, ocorreu o desenvolvimento de uma consciência crítica no que diz respeito à prática do Lian Gong como medida preventiva e terapêutica contra as LER/DORT. Isso nos estimulou a difundir este conhecimento em prol da construção de hábitos saudáveis de vida, cujo foco, na próxima fase do projeto, será a realização do Lian Gong em 18 terapias junto aos trabalhadores da indústria de cosméticos.

\section{CONSIDERAÇÕES FINAIS}

Este trabalho permitiu desvelar que a experiência de elaborar um projeto de extensão é um recurso bastante enriquecedor, no que diz respeito ao aprendizado no âmbito profissional e pessoal para os extensionistas.

Almejamos que os trabalhadores possam implementar práticas saudáveis no cotidiano das suas atividades laborais e que, a exemplo do Lian Gong em 18 terapias, consigam, com essas práticas, tratar problemas, prevenir o aparecimento de doenças e promover a saúde laboral, intervindo diretamente sobre os determinantes de vida e trabalho e proporcionando melhor qualidade de vida aos seus praticantes.

\section{Referências}

1. Ministério da Saúde (BR). Notificações estaduais de agravos relacionados ao trabalho [Internet]. 2009. [citado em 2012 Mai 22] Disponível em: http://portal. saude.gov.br/portal/saude/visualizar_texto.cfm?idtxt=29734\&janela=1.

2. Severo C, Pezzini G, Cattelan AV. Lesões por esforços repetitivos/Distúrbios osteomusculares relacionados ao trabalho (LER/DORT): a mais nova epidemia na saúde pública brasileira [Internet]. 2006. [citado em 2012 Mai 26]. Disponível em: http://www.wgate.com.br/conteudo/medicinaesaude/fisioterapia/traumato/ ler_dort_anderson.htm

3. Siqueira ACA. Os significados atribuídos às LER/DORT na relação médico-paciente: um estudo entre médicos peritos do INSS/SP e pacientes portadores de LER/DORT usuários do CEREST/SP [tese]. 2007. [citado em 2012 Jun 05]. Disponível em http://www.teses.usp.br/teses/disponiveis/5/5137/tde-11032008142450/pt-br.php

4. Maeno M, Carmo JC. Saúde do trabalhador no SUS: aprender com o passado, trabalhar o presente, construir o futuro. São Paulo (SP): Hucitec; 2005.

5. Brasil. Lei 8.213 de 24 de julho de 1991. Dispõe sobre os Planos de Benefícios da Previdência Social e dá outras providências [Internet]. 1991. [citado em 2012 Jun 05]. Disponível em http://www.planalto.gov.br/ccivil_03/leis/L8213cons.htm 6. Maffacciolli R. Os grupos na atenção básica de saúde de Porto Alegre: uso e modos de intervenção terapêutica [dissertação]. 2007. [citado em 2011 Ago 23]. Disponivel em: http://www.bibliotecadigital.ufrgs.br/da.php?nrb=000600294\&lo $c=2007 \&$ \& $=4$ de25094c034b2f1

7. Cintra MER, Pereira PPG. Percepções de corpo identificadas entre pacientes e profissionais de medicina tradicional chinesa do Centro de Saúde Escola do Butantã [Internet]. 2012. [citado em 2012 Jun 23]. Disponível em: http://www. scielo.br/scielo.php?script=sci_arttext\&pid=S0104-12902012000100019\&lang=pt 8. Bettarello PA, Saut TB. Análise dos resultados do questionário sobre qualidade de vida - SF36, após aplicação do Lian Gong em 18 terapias e de ginástica laboral, em funcionários do setor de editoração do Centro Universitário Claretiano de Batatais - SP [monografia]. Batatais: Centro Universitário Claretiano de Batatais; 2006.

9. Gouveia R. Efeito de um programa de ginástica laboral baseado na metodologia de Lian Gong em 18 terapias - um estudo realizado em trabalhadores administrativos de uma unidade de cuidados de saúde [dissertação]. 2011. [citado em 2012 Mar 23]. Disponível em: http://repositorioaberto.up.pt/ bitstream/10216/57172/2/Tese\%20Rafaela\%20Gouveia.pdf.

10. Livramento G, Franco T, Livramento A. A ginástica terapêutica e preventiva chinesa Lian Gong/Qi Gong com um dos instrumentos na prevenção e reabilitação das LER/DORT [Internet]. 2010 [citado em 2012 Mar 22]. Disponível em: http://redalyc.uaemex.mx/redalyc/pdf/1005/100513733009.pdf. 11. Brasil. Práticas Integrativas e Complementares em Saúde: Uma Realidade no SUS [Internet]. 2008. [citado em 2011 Ago 19]. Disponível em: http://portal.saude. gov.br/portal/arquivos/pdf/revcapa3.pdf.

12. Souza LMS, Rodrigues DMO, Caron CV. A utilização da técnica corporal chinesa Lian Gong no estágio supervisionado I e II do curso de naturologia aplicada da Universidade do Sul de Santa Catarina - UNISUL. Cad Acadêm. 2011;3(2):50-72.

13. Siviero EK. O papel da ginástica terapêutica chinesa Lian Gong em 18 terapias no comportamento psicomotor e cotidiano de praticantes adultos [dissertação]. Rio Claro: Universidade Estadual Paulista; 2004.

14. Silva RL, Pásztor MS. Sociedade Paulista de Lian Gong e Filosofia Oriental [Internet]. 2008. [citado em 2012 Mai 23]. Disponível em: http://www.liangong. com.br.

15. Moreira MRC, Sousa, ASA, Dias FL, Nascimento FF. Reflexões sobre a prática do Lian Gong em saúde coletiva. In: III Encontro Universitário da Universidade Federal do Ceará, Campus Cariri; 2011 out 25-27; Ceará, Brasil. 